\title{
TRANSLATES ARE ALWAYS DENSE ON THE HALF LINE
}

\author{
DONALD J. NEWMAN ${ }^{1}$
}

Let $f(x)$ be a nontrivial function in $L^{1}(-\infty, \infty)$. The celebrated theorem of Wiener tells us exactly when the linear combinations of the translates of this function are dense in $L^{1}(-\infty, \infty)$. [Namely, when the Fourier transform of $f$ has no zeros.] Suppose we ask, however, when these translates are dense in $L^{1}(0, \infty)$. The surprising answer is: always.

Similar remarks hold for the other $L^{p}$ classes and also for some sets slightly larger than the half line but we omit the details. We prove only the

THEOREM. If $f(x)$ is any nontrivial function in $L^{1}(-\infty, \infty)$ then the translates of $f(x)$ span all of $L^{1}(0, \infty)$.

The first step of the proof is Wiener's "localization" lemma, see [1]. Writing $\hat{f}(x)$ for the Fourier transform of $f(x)$ and introducing the usual "triangle" function $T_{\xi, \delta}(x)=(\delta-|x-\xi|)_{+}$we have

LeMma 1. If $\hat{f}(x)$ has no zeros in $[a, b]$ then for sufficiently small $\delta>0$ and all $\xi$ in $[a+\delta, b-\delta]$ the quotient $T_{\xi, \delta}(x) / \hat{f}(x)$ is the Fourier transform of an $L^{1}(-\infty, \infty)$ function.

We also need the following simple lemma

LEMMA 2. If $F(x)$ is $L^{1}(0, \infty)$ and non-0 a.e. and if $\xi$ varies through a set of positive measure then the collection of functions $\left\{F(x) e^{i \xi x}\right\}$ spans $L^{1}(0, \infty)$.

Proof. Suppose $G(x) \in L^{\infty}(0, \infty)$ were orthogonal to each of the $F(x) e^{i \xi x}$, that is that $\int_{0}^{\infty} G(x) F(x) e^{i \xi x} d x=0$ for all such $\xi$. The function defined by $\phi(z)=\int_{0}^{\infty} G(x) F(x) e^{i z x} d x$ is clearly analytic in $\operatorname{Im} z>0$ and continuous in $\operatorname{Im} z \geqq 0$. Vanishing on a set of positive measure is impossible for the boundary values of such an analytic function unless the function vanished identically. Thus $\phi(z) \equiv 0$ and so $F(x) G(x)=0$ a.e., and so $G(x)=0$ a.e. This proves that our collection does indeed span. Our theorem now follows easily. By Lemma 1 we have $T_{\xi, \varepsilon}(x)$ $=\hat{f}(x) \hat{g}(x)$ for some $g \in L^{1}(-\infty, \infty)$ and so, taking inverse transforms, we have

$$
e^{i \xi x} \frac{\sin ^{2} \delta x}{\pi x^{2}}=\int_{-\infty}^{\infty} f(x+t) g(-t) d t .
$$

Received by the editors August 10, 1968.

${ }^{1}$ Partially supported by the National Research Council of Canada during a visit to the University of Alberta. 
Since the right-hand side is clearly spanned by translates of $f$ we conclude that all the functions $e^{i \xi x} F(x)$ are so spanned for $\xi \in[a+\delta, b-\delta], F(x)=\sin ^{2} \delta x / \pi x^{2}$. In turn these functions span $L^{1}(0, \infty)$ by Lemma 2 and the proof is complete.

\section{REFERENCE}

1. G. H. Hardy, Divergent series, Clarendon Press, Oxford, 1949, pp. 290-291.

Belfer Graduate School of Science, Yeshiva University 